\title{
$\begin{array}{ll}\text { Research Square } & \text { Preprints are preliminary reports that have not undergone peer review. } \\ \text { They should not be considered conclusive, used to inform clinical practice, } & \text { or referenced by the media as validated information. }\end{array}$
}

\section{A prehospital diagnostic algorithm for strokes using machine learning: a prospective observational study}

\section{Yosuke Hayashi}

Department of Emergency and Critical Care Medicine, Chiba University Graduate School of Medicine

\section{Tadanaga Shimada}

Department of Emergency and Critical Care Medicine, Chiba University Graduate School of Medicine Noriyuki Hattori

Department of Emergency and Critical Care Medicine, Chiba University Graduate School of Medicine

Takashi Shimazui

Department of Emergency and Critical Care Medicine, Chiba University Graduate School of Medicine

Yoichi Yoshida

Department of Neurological Surgery, Chiba University Graduate School of Medicine

Rie E. Miura

Smart119 Inc.

Yasuo Yamao

Smart119 Inc.

Ryuzo Abe

Department of Emergency and Critical Care Medicine, Chiba University Graduate School of Medicine Eiichi Kobayashi

Department of Neurological Surgery, Chiba University Graduate School of Medicine

\section{Yasuo Iwadate}

Department of Neurological Surgery, Chiba University Graduate School of Medicine

Taka-aki Nakada ( $\nabla$ taka.nakada@nifty.com )

Department of Emergency and Critical Care Medicine, Chiba University Graduate School of Medicine

\section{Research Article}

Keywords: prediction, acute ischemic stroke, subarachnoid hemorrhage, intracerebral hemorrhage, XGBoost

Posted Date: May 13th, 2021

DOI: https://doi.org/10.21203/rs.3.rs-492777/v1

License: (c) (i) This work is licensed under a Creative Commons Attribution 4.0 International License. 
Page 2/16 


\section{Abstract}

High precision is optimal in prehospital diagnostic algorithms for strokes and large vessel occlusions (LVOs). We hypothesized that prehospital diagnostic algorithms for strokes and their subcategories using machine learning could have high predictive value. Consecutive adult patients with suspected stroke as per emergency medical service personnel were enrolled in a prospective multicenter observational study in 12 hospitals in Japan. Five diagnostic algorithms using machine learning, including logistic regression, random forest, support vector machine, and eXtreme Gradient Boosting (XGBoost), were evaluated for stroke and subcategories including acute ischemic stroke (AIS) with/without LVO, intracranial hemorrhage $(\mathrm{ICH})$, and subarachnoid hemorrhage (SAH). Of the 1446 patients in the analysis, $1156(80 \%)$ were randomly included in the training cohort and $290(20 \%)$ were included in the test cohort. In the diagnostic algorithms for strokes using XGBoost had the highest diagnostic value (test data, area under the receiver operating curve [AUROC] 0.980, confidence interval [Cl; 0.962-0.994]). In the diagnostic algorithms for the subcategories using XGBoost had a high predictive value (test data, AUROC [CI], AIS with LVO 0.898 [0.848-0.939], AIS without LVO 0.882 [0.836-0.923], ICH 0.866 [0.817-0.911], SAH 0.926 [0.874-0.971]). Prehospital diagnostic algorithms using machine learning had high predictive value for strokes and their subcategories.

\section{Introduction}

Stroke is an acute life-threatening disease that mostly occurs in out-of-hospital settings ${ }^{1}$. Emergency medical services (EMS) personnel, who are the first healthcare providers to respond to patients with a suspected stroke, evaluate the risk of stroke and transport those patients to hospitals. Early initiation of therapeutic approaches, including endovascular therapy for large vessel occlusion (LVO) ${ }^{2,3}$ is key to improving the clinical outcomes of strokes ${ }^{4}$. Thus, higher precision of stroke prediction in prehospital settings may contribute to improving the quality of stroke care and clinical outcomes.

Prehospital diagnostic algorithms for strokes, which are also known as stroke scales, have been developed substantially ${ }^{5-7}$. However, there is room for improvement in the prediction precision for strokes or LVOs (area under the receiver operating characteristic curve [AUROC], stroke prediction, Cincinnati

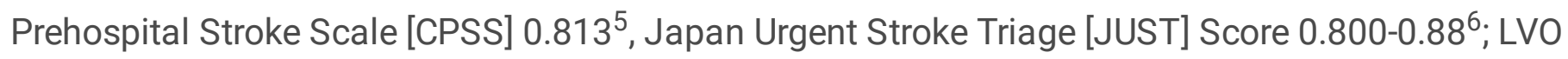
prediction, 8 prehospital stroke scales $\left.0.72-0.83^{7}\right)$. Recent advances in statistical approaches using machine learning have significantly improved the precision of predicting algorithms for acute diseases, including out-of-hospital cardiac arrest, acute coronary syndrome, and sepsis ${ }^{8-10}$. However, few investigations have focused on prehospital stroke/LVO diagnostic algorithms using machine learning.

Therefore, we tested the hypothesis that prehospital stroke diagnostic algorithms using machine learning had a high predictive value. We prospectively enrolled a large cohort of patients with suspected stroke in prehospital settings and analyzed them using five machine learning algorithms. 


\section{Results}

\section{Baseline characteristics and outcomes}

Of the 1446 patients analyzed, $1156(80 \%)$ were randomly included in a training cohort. Of these, 834 patients had a stroke (training cohort, Table 1). Patients with strokes had significantly increased age, increased past history of heart diseases (atrial fibrillation and hypertension), decreased past history of diabetes mellitus, and decreased past history of neurological diseases compared to non-stroke patients. In terms of vital signs, patients with strokes had decreased heart rates, increased arrhythmia, increased blood pressure, and decreased probability of impaired Glasgow coma scale (GCS) components compared to non-stroke patients. Patients with strokes had decreased dizziness and convulsion and increased upper limb paralysis, hemiparalysis, facial palsy, and dysarthria compared to non-stroke patients. Onset timing (hourly) was earlier in patients with strokes than in those without strokes. Similar differences were observed in the test cohort data (test cohort, Supplementary Table S2). 
Table 1

Baseline characteristics and clinical outcomes in the training cohort

\begin{tabular}{|c|c|c|c|}
\hline & Stroke $(n=834)$ & Non-stroke $(n=322)$ & $P$ value \\
\hline Age, years & $74.0(65.0-82.0)$ & $72.0(57.2-81.0)$ & .004 \\
\hline Male sex, n (\%) & $507(60.8)$ & $182(56.5)$ & .208 \\
\hline \multicolumn{4}{|l|}{ Past medical history } \\
\hline Atrial fibrillation, n (\%) & $70(8.4)$ & $14(4.3)$ & .003 \\
\hline Hypertension, n (\%) & $380(45.6)$ & $138(42.9)$ & .017 \\
\hline Diabetes mellitus, n (\%) & $109(13.1)$ & $53(16.5)$ & .015 \\
\hline Intracranial hemorrhage, n (\%) & $38(4.6)$ & $27(8.4)$ & $<.001$ \\
\hline Cerebral infarction, $\mathrm{n}(\%)$ & $157(18.8)$ & $62(19.3)$ & .008 \\
\hline Epilepsy, n (\%) & $6(0.7)$ & $13(4.0)$ & $<.001$ \\
\hline Psychiatric disorder, n (\%) & $21(2.5)$ & $23(7.1)$ & $<.001$ \\
\hline \multicolumn{4}{|l|}{ Vital signs } \\
\hline Heart rate & $82(70-96)$ & $84(74-98)$ & .033 \\
\hline Arrhythmia, n (\%) & $192(23.0)$ & $37(11.5)$ & $<.001$ \\
\hline Systolic blood pressure & $174(155-200)$ & $160(140-180)$ & $<.001$ \\
\hline Diastolic blood pressure & $97(83-114)$ & $90(79-104)$ & $<.001$ \\
\hline Body temperature & $36.5(36.2-36.8)$ & $36.5(36.2-36.8)$ & .725 \\
\hline Japan Coma Scale = 0, n (\%) & $357(42.8)$ & $170(52.8)$ & .007 \\
\hline \multicolumn{4}{|l|}{ Glasgow Coma Scale } \\
\hline Eye opening = 4, n (\%) & $627(75.2)$ & $269(83.5)$ & .019 \\
\hline Best verbal response $=5, \mathrm{n}(\%)$ & $402(48.2)$ & $188(58.4)$ & .003 \\
\hline Best motor response $=6, \mathrm{n}(\%)$ & $608(72.9)$ & $257(79.8)$ & .018 \\
\hline \multicolumn{4}{|l|}{ Symptoms } \\
\hline Vomiting, n (\%) & $135(16.2)$ & $38(11.8)$ & .114 \\
\hline
\end{tabular}

JCS (Japan coma scale), GCS (Glasgow coma scale), THI (thermo-hydrological index).

Data are presented as median and interquartile range for continuous variables.

$P$-values were calculated using Pearson's chi-square test or the Mann-Whitney U test. 


\begin{tabular}{|c|c|c|c|}
\hline & Stroke $(n=834)$ & Non-stroke $(n=322)$ & $P$ value \\
\hline Dizziness, n (\%) & $49(5.9)$ & $46(14.3)$ & $<.001$ \\
\hline Convulsion, n (\%) & $15(1.8)$ & $40(12.4)$ & $<.001$ \\
\hline Upper limbs paralysis, n (\%) & $354(42.4)$ & $115(35.7)$ & .043 \\
\hline Lower limbs paralysis, n (\%) & $431(51.7)$ & $152(47.2)$ & .194 \\
\hline Hemiparalysis, n (\%) & $198(23.7)$ & $32(9.9)$ & $<.001$ \\
\hline Conjugate deviation, n (\%) & $90(10.8)$ & $21(6.5)$ & .064 \\
\hline Visual field defects, n (\%) & $14(1.7)$ & $4(1.2)$ & .228 \\
\hline Facial palsy, n (\%) & $55(26.3)$ & $10(12.3)$ & .036 \\
\hline Ataxia, n (\%) & $23(11.0)$ & $8(9.9)$ & .824 \\
\hline Sensory impairment, $\mathrm{n}(\%)$ & $29(13.9)$ & $4(4.9)$ & .066 \\
\hline Aphasia, n (\%) & $69(33.0)$ & $22(27.2)$ & .411 \\
\hline Dysarthria, n (\%) & $69(33.0)$ & $11(13.6)$ & .001 \\
\hline Unilateral spatial neglect, $\mathrm{n}(\%)$ & $29(3.5)$ & $6(1.9)$ & .138 \\
\hline Onset timing Monday, n (\%) & $136(16.3)$ & $40(12.4)$ & .120 \\
\hline Onset timing (hour) & $12(7-18)$ & $14(8-19)$ & .023 \\
\hline Minimum THI & $13.1(7.7-18.7)$ & $12.2(8.2-18.8)$ & .849 \\
\hline \multicolumn{4}{|c|}{ JCS (Japan coma scale), GCS (Glasgow coma scale), THI (thermo-hydrological index). } \\
\hline \multicolumn{4}{|c|}{ Data are presented as median and interquartile range for continuous variables. } \\
\hline \multicolumn{4}{|c|}{$P$-values were calculated using Pearson's chi-square test or the Mann-Whitney U test. } \\
\hline
\end{tabular}

\section{Prediction of stroke}

In the primary analysis of stroke prediction using logistic regression, random forest, support vector machines (SVM), and eXtreme Gradient Boosting (XGBoost), XGBoost had the highest predictive values (AUROC 0.994 [confidence interval; $\mathrm{Cl} 0.991-0.997$ ]) in the training cohort. In the test cohort, the XGBoost model also had the highest predictive value of the five machine learning approaches (AUROC 0.980 [CI 0.962-0.994]) (Table 2 and Figure a, b). The SHapley Additive exPlanation (SHAP) summary plot revealed that the major predictive contributors for stroke were "sudden headache," "upper limb paralysis," "convulsion," "sudden impaired consciousness or headache," "systolic blood pressure," "arrhythmia," "conjugate deviation," and "diastolic blood pressure" (Figure c). 
Table 2

Prehospital stroke prediction using machine learning

\begin{tabular}{|llllll|}
\hline Models & AUROC & Accuracy & Sensitivity & Specificity & F1-score \\
\hline Training cohort & & & & & \\
\hline XGBoost & 0.994 & 0.978 & 0.990 & 0.947 & 0.985 \\
\hline Random forest & 0.979 & 0.943 & 0.956 & 0.910 & 0.960 \\
\hline SVM (Radial basis function) & 0.968 & 0.928 & 0.950 & 0.873 & 0.950 \\
\hline SVM (Linear) & 0.889 & 0.835 & 0.915 & 0.627 & 0.889 \\
\hline Logistic regression & 0.882 & 0.843 & 0.847 & 0.835 & 0.886 \\
\hline Test cohort & & & & & 0.967 \\
\hline XGBoost & 0.980 & 0.952 & 0.986 & 0.864 & 0.935 \\
\hline Random forest & 0.953 & 0.907 & 0.933 & 0.840 & 0.931 \\
\hline SVM (Radial Basis function) & 0.935 & 0.900 & 0.933 & 0.815 & 0.907 \\
\hline SVM (Linear) & 0.904 & 0.862 & 0.928 & 0.691 & 0.874 \\
\hline Logistic regression & 0.886 & 0.828 & 0.828 & 0.827 & \\
\hline $\begin{array}{l}\text { AUROC (area under the receiver operating characteristic curve), XGBoost (eXtreme Gradient Boosting) } \\
\text { SVM (support vector machine) }\end{array}$ & & & & \\
\hline
\end{tabular}

\section{Prediction of stroke subcategories}

We next analyzed the prediction algorithms for stroke subcategories (AIS with / without LVO, ICH, and SAH) using XGBoost, which was the best approach in the primary analysis. The machine learning-based prediction algorithms had high predictive values (test data, AUROC [CI], AIS with LVO 0.898 [0.848-0.939], AIS without LVO 0.882 [0.836-0.923], ICH 0.866 [0.817-0.911], SAH 0.926 [0.874-0.971]) (Table 3 and Supplementary Figure S2). The SHAP summary plot of AIS with LVO revealed that the major contributors were "GCS V," "onset hours," "age," "arrhythmia," "hemiparalysis," "systolic blood pressure," "Japan Coma Scale (JCS)," and "minimum thermo-hydrological index (THI)" (Supplementary Figure S2). 
Table 3

Prehospital stroke subcategory prediction using XGBoost

\begin{tabular}{|llllll|}
\hline & AUROC & Accuracy & Sensitivity & Specificity & F1-score \\
\hline Training cohort & & & & \\
AIS with LVO & 0.896 & 0.893 & 0.384 & 0.977 & 0.504 \\
AIS without LVO & 0.916 & 0.837 & 0.840 & 0.835 & 0.736 \\
ICH & 0.910 & 0.853 & 0.679 & 0.906 & 0.684 \\
SAH & 0.974 & 0.971 & 0.574 & 0.993 & 0.673 \\
Test cohort & & & & 0.964 \\
AIS with LVO & 0.898 & 0.897 & 0.488 & 0.571 \\
AIS without LVO & 0.882 & 0.814 & 0.810 & 0.815 & 0.703 \\
ICH & 0.866 & 0.834 & 0.618 & 0.901 & 0.636 \\
$\begin{array}{l}\text { SAH } \\
\text { SAH }\end{array}$ & 0.926 & 0.952 & 0.333 & 0.985 & 0.417 \\
\hline $\begin{array}{l}\text { AUROC (area under the receiver operating characteristic curve), XGBoost (eXtreme Gradient Boosting), } \\
\text { SVM (support vector machine), AlS (acute ischemic stroke), LV (large vessel occlusion), ICH } \\
\text { (intracranial hemorrhage), SAH (subarachnoid hemorrhage }\end{array}$ & & \\
\hline
\end{tabular}

\section{Discussion}

In this study of prehospital stroke prediction using machine learning, the algorithm using XGBoost had a high predictive value for strokes (AUROC 0.980 [Cl 0.962-0.994]) and stroke subcategories including LVO (AUROC 0.898 [Cl 0.848-0.939]).

Substantial investigations on prehospital predicting scales for strokes have been conducted with insufficient precision ${ }^{5-7}$. In a meta-analysis of the CPSS for stroke prediction (study $n=3$, patient $n=$ 1366), the CPSS had a summary AUROC of $0.813^{5}$. More recent investigation of the JUST score (patient $\mathrm{n}=2236$ ) documented AUROCs of 0.88 and 0.80 for stroke in the derivation and validation cohorts, respectively ${ }^{6}$. In accordance with these stroke predictions, insufficient predictive values of LVO prediction (AUROC 0.72-0.83) were documented in eight prehospital scales including the Rapid Arterial Occlusion Evaluation (RACE), Los Angeles Motor Scale, Cincinnati Stroke Triage Assessment Tool, Gaze-Face-ArmSpeech-Time, Prehospital Acute Stroke Severity, CPSS, Conveniently-Grasped Field Assessment Stroke Triage, and Face-Arm-Speech-Time plus severe arm or leg motor deficit test ${ }^{7}$. Thus, there is an unmet need for the improvement of the prediction precision for strokes/LVOs. The machine learning approach is a potential solution to improve precision, as we have demonstrated in this study. We found that the prediction algorithms using XGBoost had a high predictive value for strokes (AUROC 0.980) and stroke subcategories (AUROC 0.866-0.926) including LVOs (AUROC 0.898). 
Substantial machine learning studies for diagnosis and prognosis in acute diseases have been documented $^{8-10}$, while machine learning studies for strokes remain insufficient. To the best of our knowledge, investigations on predicting strokes by machine learning using a prehospital dataset have rarely been conducted. The majority of machine learning studies are focused on detecting strokes from computed tomography (CT) images, and machine learning studies focused on prehospital or hospital bedside prediction of strokes are limited ${ }^{11}$. In this study, we successfully developed prehospital stroke prediction algorithms using a machine learning approach with high precision. We found only one machine learning study that predicted LVOs with prehospital data. The study used 24 variables including prehospital data on 777 adult AIS patients (LVO $n=300)$ who underwent CT angiography or MR angiography and received reperfusion therapy within 8 hours from symptom onset in a single center. They compared artificial neural network (ANN) and conventional stroke prediction scales including the Cincinnati Prehospital Stroke Severity Scale, Field Assessment Stroke for Emergency Destination, and RACE. They found that the ANN had a higher predictive value than the conventional scales (AUROC, ANN 0.823 , conventional scales $0.740-0.796)^{12}$. In addition, prediction algorithms for LVOs using machine learning were documented by You et al., whose study was not a prehospital study but a hospital study ${ }^{13}$. Their study included 300 adult stroke patients (LVO $n=130)$ with 24 variables and compared the predictive values of XGBoost, logistic regression, random forest, and SVM. They found that XGBoost had the highest predictive value for LVOs (AUROC 0.809) ${ }^{13}$. In agreement with these findings, we also found that XGBoost had a higher predictive value for strokes compared to logistic regression, random forest, and SVM. Thus, XGBoost appears to be a better approach for this prediction.

Not only patients' baseline factors, but also environmental factors, increased the risk of stroke ${ }^{14}$. In this study, of the 21 environmental factors screened, the onset timing factors including onset hour, day of the week (Monday), and minimum THI were analyzed. Stroke patients had earlier onset timing hours compared to non-stroke patients in the univariate analysis (Table 1). In addition, the SHAP analysis in the XGBoost for the identified onset hour of a stroke had an impact on the algorithms. Regarding the minimum THI, a high impact was identified in the SHAP analysis for LVOs. Few prehospital stroke scales include environmental factors; however, adding environmental factors may contribute to improving precision of prehospital stroke/stroke subcategory prediction using machine learning.

There were some limitations to this study. First, the study was a multicenter study, but conducted in a single urban region in Japan. Second, we aimed for a better performance rather than limiting the number of predictors. Further studies including wider regions or a limited number of predictors may strengthen the findings for the prediction of strokes by machine learning.

In the conclusions, the prehospital stroke diagnostic algorithm using machine learning had a high predictive value for strokes and their subcategories including LVOs. This machine learning approach could potentially lead to precise stroke recognition in prehospital settings.

\section{Methods}




\section{Study setting and patients}

The current multicenter observational study was prospectively conducted in an urban area (Chiba city, population 1 million) in Japan, between September 2018 and September 2020. Consecutive adult patients ( $\geq 20$ years of age) with suspected stroke as per EMS personnel who were subsequently transported to one of 12 hospitals were enrolled. Of the 1778 patients screened, 332 who had missing diagnostic data or multiple entries were excluded and 1446 were analyzed (Supplementary Figure S1).

The study was approved by the Ethical Review Board of the Graduate School of Medicine, Chiba University (No.2733). The review board waived the need for written informed consent, in conformity with the Ethical Guidelines for Medical and Health Research Involving Human Subjects in Japan. There will be no availability of the clinical data and study materials for other research institutes to re-analysis and duplicate the results.

\section{Data collection and definition}

Data on variables for stroke prediction in the prehospital setting, including symptoms, physiological data, and medical history, were collected (Supplementary Table S1). The variables cover each of the scoring components of the National Institutes of Health Stroke Scale ${ }^{15}$. Since onset timing (daily, especially Monday, and hourly) and meteorological conditions potentially altered the risk of stroke ${ }^{14,16}$, onset time and weather data were added to the analysis.

Stroke is defined based on the National Institute of Neurological Disorders and Stroke III $^{17}$. Stroke is further subclassified into acute ischemic stroke (AIS) with LVO, AIS without LVO, intracranial hemorrhage $(\mathrm{ICH})$, and subarachnoid hemorrhage (SAH). LVO is defined as acute occlusion of the internal carotid artery, M1 or M2 portion of the middle cerebral artery, and the basilar artery. Board-certified neurologists in registered hospitals made the diagnoses based on examinations including $\mathrm{CT}$, magnetic resonance imaging (MRI), CT angiography, and MR angiography.

\section{Missing values}

We used domain knowledge to impute missing values first. The mutually imputed pairs or groups of features based on the knowledge were as follows: (i) conjugate deviation and visual field defects, (ii) dysarthria and facial paralysis, (iii) aphasia, best verbal response of the GCS, the JCS, and consciousness-related features.

For the remaining missing values of highly correlated variables (correlation coefficient $>0.7$ ), multivariate imputation was applied between the variables using a regressor model, in which each feature with missing values is modeled as a function of other features. The mutually imputed pairs or groups of features were as follows: (i) systolic and diastolic blood pressure, (ii) left and right pupil sizes, (iii) left and right pupillary light reflex, (iv) the GCS, the JCS, and consciousness-related features, and (v) paralysis-related features. Other missing values of the numerical features were imputed with each 
median value. For any other categorical attributes, the missing values were replaced with a new subcategory "Unknown."

Note that XGBoost can handle missing values, unlike the other methods. Therefore, we trained two XGBoost models, one with the data before and the other after applying imputation, and confirmed that the imputation did not cause a decrease in performance (paired sampled t-test's $P$-value $=0.268$ for 10 fold cross validation scores). For the purpose of comparison between the other models (logistic regression, random forest, SVM) and XGBoost, we used the imputed data for all analyses in this study.

\section{Feature selection}

Among the 59 features collected by EMS personnel and onset time variables, 51 features were selected after primitive cleaning of the feature candidates with a large fraction (over $40 \%$ ) of missing values (2 features) or low variance ( $5 \%$ cutoff, 6 features).

For the meteorological features, we calculated Pearson's chi-square values for all pairs between the stroke category and the 21 meteorological features (Supplementary Methods) and selected the features with pvalues $<0.05$ as feature candidates. These selected meteorological features were further reduced in number by a forward stepwise selection method, in which only features that improved the performance of the model were selected by repeatedly including the features one by one. Finally, only one meteorological feature, the 'minimum THI on the onset day' was selected.

Because we aimed to improve performance, rather than simply limit the number of predictors, we included 52 features for all analyses in this study.

\section{Statistical analysis}

First, we developed the binary classification models for stroke classification as a primary outcome based on five common machine learning algorithms: logistic regression, random forest, SVM with linear or radius basis function kernels, and XGBoost. Then, as a secondary outcome, we built a multi-class classification model to predict each stroke category of AIS with/without LVO, ICH, and SAH. Based on the primary analysis that found XGBoost to be superior, we chose XGBoost for the secondary outcome analysis.

The parameters of the five machine learning models were selected by using the grid search method, in which we further split the training cohort into five folds, trained each of the five sets of data, and selected the parameters of the model that performed the best. It should be noted that the data are imbalanced. More weight must be set toward the minor classes in any model when the loss functions are calculated so that the major and minor classes are fairly evaluated.

The performance of the models was measured in terms of the AUROC as a superior metric, as well as sensitivity, specificity, and the F1 score. We used the SHAP algorithm ${ }^{18}$ of the XGBoost model to interpret the contributions of each feature to the predictive model. In the algorithm, the SHAP value was computed by a difference in model output resulting from the inclusion of a feature in the algorithm, providing 
information on each feature's impact on the output. In the SHAP summary plots, every violin plot is composed of all data points of each feature with a higher value being redder, and a lower value being bluer. The violin plots are aligned with the SHAP value along the $x$-axis. Thus, a redder/bluer violin plot on the right side (i.e., higher positive SHAP value) suggests that the higher/lower the values of the feature are, the more the model predicts towards positive/negative impact.

Data are expressed as medians (interquartile ranges) for continuous values and absolute numbers and percentages for categorical values. Two-tailed $p$-values $<0.05$ were considered significant. Analyses were performed using Python 3.7.6 packages (Scikit-learn 0.23.2, XGBoost 1.1.1, Pandas 1.1.5, and NumPy 1.19.2) to construct the machine learning models.

\section{Declarations}

\section{Data availability}

The datasets used and analyzed during our study are available from the corresponding author upon reasonable request.

\section{Acknowledgements}

We thank all contributors to this study, especially the following investigators: Chiba Emergency Medical Center (Akihiro Miyata), Chiba Medical Center (Iwao Yamakami), Chiba Chuo Medical Center (Motoki Sato), Chiba Neurosurgical Hospital (Takuji Igarashi), National Hospital Organization Chiba Medical Center (Hiroaki Ozaki), Chiba Aoba Municipal Hospital (Yasumasa Morita), Mitsuwadai General Hospital (Masahiko Kasai), Japan Community Health Care Organization Chiba Hospital (Noriyoshi Murotani), Sannou Hospital (Tune Yajima), Kashiwado Hospital (Toshihiro Saito), Kajita medical clinic (Masahito Kajita), and Chiba City Fire Department (Hideki Shinhama).

\section{Author contributions}

Study concept and design: TN, RA, YY (Yoshida), YY (Yamao)

Acquisition of data: $\mathrm{TN}, \mathrm{NH}, \mathrm{TS}$ (Shimada), $\mathrm{YH}$

Drafting of the manuscript: TN, TS (Shimada), YH, RM

Critical revision of the manuscript for important intellectual content: $\mathrm{YH}, \mathrm{TS}$ (Shimada), NH, TS (Shimazui), YY (Yoshida), RM, YY (Yamao), RA, EK, YI, TN

Statistical analysis: TN, TS (Shimada), YH, YY (Yamao), RM

Supervision: TN

All authors have read and approved the final manuscript. 
Additional Information

\section{Competing interests}

The authors have disclosed that they do not have any conflicts of interest.

\section{Source of Funding}

Taka-aki Nakada is currently receiving a grant (FDN \#JPhe1502001) from "the Japan Agency for Medical Research and Development". The funder had no role in the study design, experiments, collection of data, analyses, interpretation of data, writing of the manuscript, or decision to submit the manuscript for publication.

TN and YY (Yamao) are inventors and have submitted patents related to this work. TN and YY (Yamao) serve as directors and hold shares in Smart119 Inc. REM serves as a chief scientist in Smart119 Inc.

\section{References}

1. Farzaneh $\mathrm{H}$. et al. A multicentre observational study of presentation and early assessment of acute stroke. BMJ. 325, 17 (2002).

2. Berkhemer O. A. et al. A randomized trial of intraarterial treatment for acute ischemic stroke. $N$ Engl $J$ Med. 372, 11-20 (2015).

3. Goyal M. et al. Randomized assessment of rapid endovascular treatment of ischemic stroke. $N$ Engl J Med. 372, 1019-1030 (2015).

4. Saver J. L. et al. Time to treatment with endovascular thrombectomy and outcomes from ischemic stroke: A meta-analysis. JAMA. 316, 1279-1288 (2016).

5. Ethan S. B., Mohit S., Richard H. S. \& Steven R.L. Prehospital stroke scales in urban environments a systematic review. Neurology. 82, 2241-2249 (2014).

6. Uchida K. et al. Clinical prediction rules to classify types of stroke at prehospital stage. Stroke. 49, 1820-1827 (2018).

7. Duvekot M. H. C. et al. Comparison of eight prehospital stroke scales to detect intracranial largevessel occlusion in suspected stroke (presto): A prospective observational study. The Lancet Neurology. 20, 213-221. (2021).

8. Seki T., Tamura T.. \& Suzuki M., Group S-KS. Outcome prediction of out-of-hospital cardiac arrest with presumed cardiac aetiology using an advanced machine learning technique. Resuscitation. 141, 128135 (2019).

9. Than M. P. et al. Machine learning to predict the likelihood of acute myocardial infarction. Circulation. 140, 899-909 (2019).

10. Seymour C. W. et al. Derivation, validation, and potential treatment implications of novel clinical phenotypes for sepsis. JAMA. 321, 2003-2017 (2019). 
11. Sirsat M. S., Ferme E. \& Camara J. Machine learning for brain stroke: A review. J Stroke Cerebrovasc Dis. 29, 105162 (2020).

12. Chen Z. et al. Novel prehospital prediction model of large vessel occlusion using artificial neural network. Front Aging Neurosci. 10, 181 (2018).

13. You J. et al. Automated hierarchy evaluation system of large vessel occlusion in acute ischemia stroke. Front Neuroinform. 14, 13 (2020).

14. Mukai T. et al. Various meteorological conditions exhibit both immediate and delayed influences on the risk of stroke events: The hews-stroke study. PLoS One. 12, e0178223 (2017).

15. Brott. T. et al. V. Measurements of acute cerebral infarction a clinical examination scale. Stroke. 20, 864-870 (1989).

16. Shigematsu K., Watanabe Y. \& Nakano H., Kyoto Stroke Registry C. Weekly variations of stroke occurrence: An observational cohort study based on the kyoto stroke registry, japan. BMJ Open. 5 , e006294 (2015).

17. Special report from the national institute of neurological disorders and stroke. Classification of cerebrovascular diseases iii. Stroke. 1990;21:637-676.

18. Lundberg S. M. et al. From local explanations to global understanding with explainable ai for trees. Nat Mach Intell. 2, 56-67 (2020).

19. Sueda Y. et al. Effects of meteorological conditions on the risk of ischemic stroke events in patients treated with alteplase-hews-tpa. J Stroke Cerebrovasc Dis. 24, 1500-1505 (2015).

\section{Figures}


a

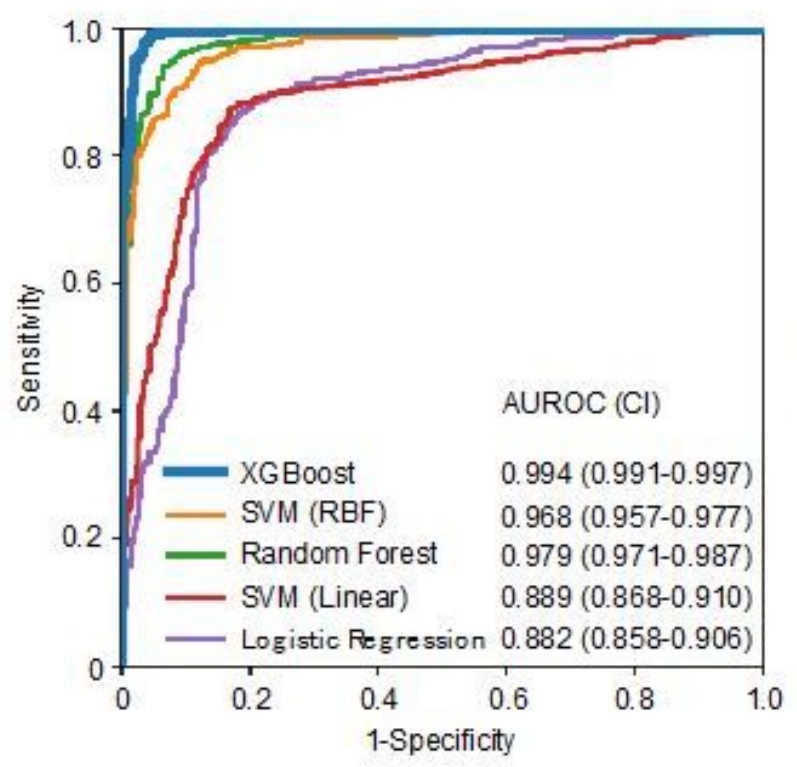

c

Sudden headache

Upper limb paralysis

Convulsion

Sudden impaired consciousness or headache

Systolic blood pressure

Arrhythmia

Conjugate deviation

Diastolic blood pressure

Facial paralysis

Lower limb paralysis

GCS M

Heart rate

Onset hour

Hemiparalysis

Dysarthria

Vomiting

Consciousness

Past medical history (Hypertension)

Age

Past medical history (Intracranial hemorrhage) b
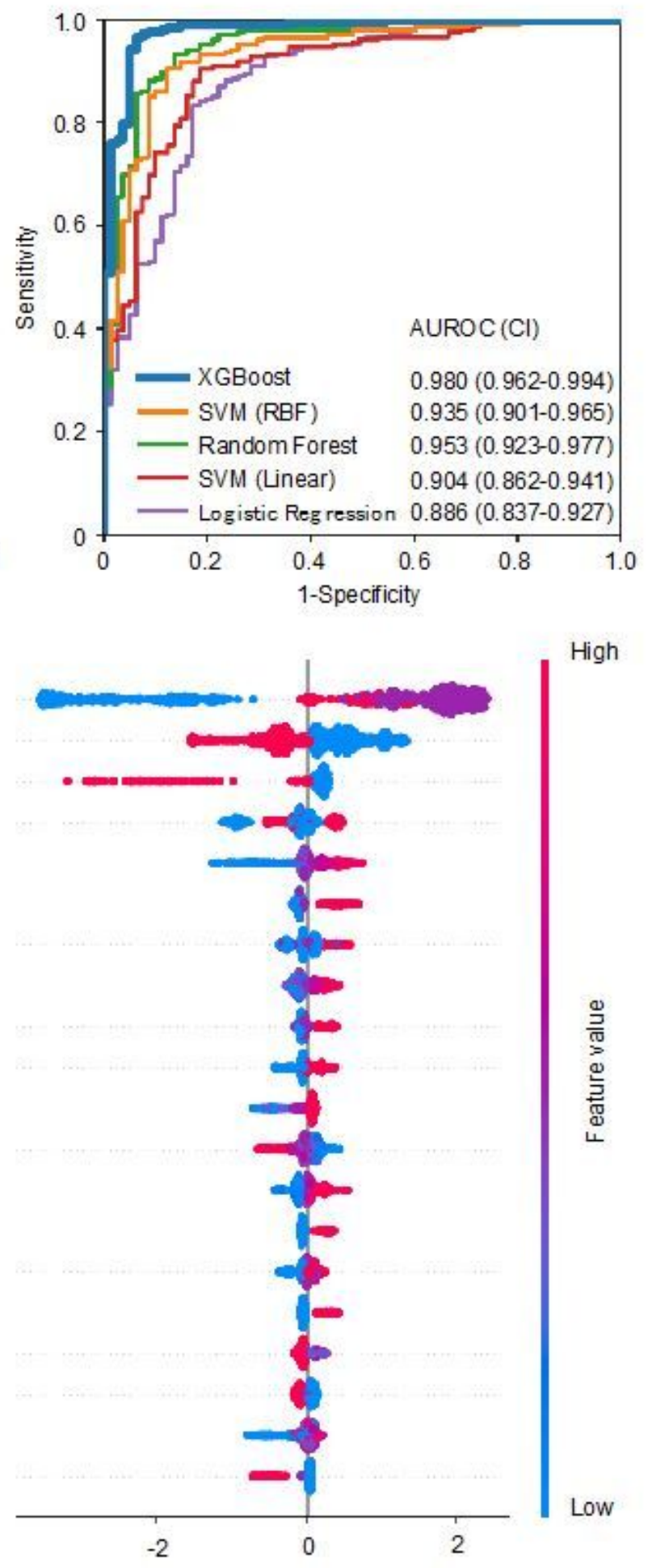

\section{Figure 1}

Receiver operating characteristic curve and the SHAP value of prehospital stroke prediction.

\section{Supplementary Files}

This is a list of supplementary files associated with this preprint. Click to download. 
- Supplementalmaterials20210510.pdf

- Supplementalmaterials20210510.pdf 